\title{
Blank Node Matching and RDF/S Comparison Functions
}

\author{
Yannis Tzitzikas, Christina Lantzaki, and Dimitris Zeginis` \\ Computer Science Department, University of Crete, \\ Institute of Computer Science, FORTH-ICS, Greece \\ \{tzitzik,kristi, zeginis\}@ics.forth.gr
}

\begin{abstract}
In RDF, a blank node (or anonymous resource or bnode) is a node in an RDF graph which is not identified by a URI and is not a literal. Several RDF/S Knowledge Bases (KBs) rely heavily on blank nodes as they are convenient for representing complex attributes or resources whose identity is unknown but their attributes (either literals or associations with other resources) are known. In this paper we show how we can exploit blank nodes anonymity in order to reduce the delta (diff) size when comparing such KBs. The main idea of the proposed method is to build a mapping between the bnodes of the compared $\mathrm{KBs}$ for reducing the delta size. We prove that finding the optimal mapping is NP-Hard in the general case, and polynomial in case there are not directly connected bnodes. Subsequently we present various polynomial algorithms returning approximate solutions for the general case.

For making the application of our method feasible also to large KBs we present a signature-based mapping algorithm with $n \log n$ complexity. Finally, we report experimental results over real and synthetic datasets that demonstrate significant reductions in the sizes of the computed deltas. For the proposed algorithms we also provide comparative results regarding delta reduction, equivalence detection and time efficiency.
\end{abstract}

\section{Introduction}

The ability to compute the differences that exist between two RDF/S Knowledge Bases (KBs) is an important step to cope with the evolving nature of the Semantic Web (SW). In particular, RDF/S Deltas can be employed to aid humans understand the evolution of knowledge, and to reduce the amount of data that need to be exchanged and managed over the network in order to build SW synchronization [191, versioning [7/81421] and replication [17] services. A rather peculiar but quite flexible feature of RDF is that it allows the representation of unnamed nodes, else called blank nodes (for short bnodes), a feature that is convenient for representing complex attributes (e.g. an attribute address as shown in Figure 10 without having to name explicitly the auxiliary node that is used

\footnotetext{
* Current affiliation: Information Systems Lab,University of Macedonia, Thessaloniki, Greece, zeginis@uom.gr.
}

P. Cudré-Mauroux et al. (Eds.): ISWC 2012, Part I, LNCS 7649, pp. 591-607, 2012.

(C) Springer-Verlag Berlin Heidelberg 2012 


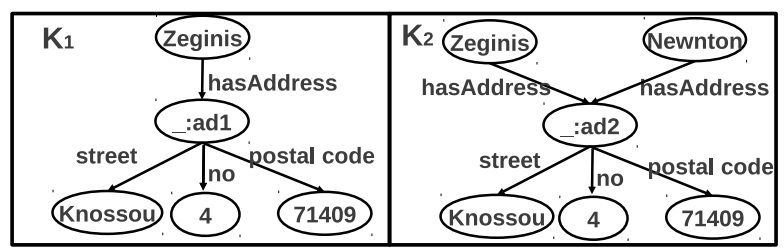

Fig. 1. Examples of blank nodes

for connecting together the values that constitute the complex value (i.e. the particular street, number and postal code values). A recent paper [10] that surveys the treatment of bnodes in RDF data, proves that blank nodes is an inevitable reality. Just indicatively, and according to their results, the data fetched from the "hi5.com" domain consist of $87.5 \%$ of blank nodes, while those from the "opencalais.com" domain, which is part of LOD (Linked Open Data) cloud, has $44.9 \%$ bnodes. The authors also state that the inability to match bnodes increases the delta size and does not assist in detecting the changes between subsequent versions of a KB.

Previous works on comparing RDF KBs have not elaborated on this issue thoroughly. There are works (e.g. 2122] proposing differential functions that yield reduced in size deltas (in certain cases) but treat bnodes as named nodes. Other works and systems (specifically Jena [3]) focus only on deciding whether two KBs that contain bnodes are equivalent or not, and do not offer any delta size saving for the case where the involved KBs are not equivalent. In brief, and to the best of our knowledge, there is not any work that attempts to establish a bnode mapping for reducing the delta size for the case of non equivalent KBs. Note that finding such a mapping can be considered as a preprocessing step, a task that is carried out before a differential function (like those described in [17/2016 158 21]) is applied.

We prove that finding the optimal mapping is NP-Hard in the general case and polynomial if there are not directly connected bnodes. Subsequently we present various polynomial algorithms returning approximate solutions for the general case. For making the application of this method feasible also to large KBs one of these algorithms has $n \log n$ complexity.

The experimental results over real and synthetic datasets showed that our method significantly reduces the sizes of the computed deltas, while the time required is affordable (just indicatively the $n \log n$ algorithm requires a few seconds for KBs with up to 150,000 bnodes). For the proposed algorithms we also provide comparative results regarding time efficiency and their potential for delta reduction and equivalence detection.

The rest of this paper is organized as follows. Section 2 discusses RDF KBs with bnodes and the equivalence of such KBs. Section 3 elaborates on the problem of finding the optimal mapping. Section 4 proposes bnode matching algorithms and Section 5 reports experimental results. Section 6 discusses the applicability of the method at the presence of inference rules and various 
semantics, Section 7 discusses related work, and finally, Section 8 concludes the paper and identifies issues for further research.

Software and datasets are available to download and use from http://www.ics.forth.gr/isl/BNodeDelta.

\section{RDF KBs with Blank Nodes}

Consider there is an infinite set $U$ (RDF URI references), an infinite set $B$ (blank nodes) and an infinite set $L$ (literals). A triple $(s, p, o) \in(U \cup B) \times U \times(U \cup B \cup L)$ is called an RDF triple ( $s$ is called the subject, $p$ the predicate and $o$ the object). An RDF Knowledge Base (KB) $K$, or equivalently an $R D F$ graph $G$, is a set of RDF triples.

For an RDF Graph $G_{1}$ we shall use $U_{1}, B_{1}, L_{1}$ to denote the URIs, bnodes and literals that appear in the triples of $G_{1}$ respectively. The nodes of $G_{1}$ are the values that appear as subjects or objects in the triples of $G_{1}$.

The equivalence of $R D F$ graphs that contain blank nodes is defined in [9] as:

\section{Def. 1 (Equivalence of RDF Graphs that contain Bnodes)}

Two RDF graphs $G_{1}$ and $G_{2}$ are equivalent if there is a bijection 11 between the sets of nodes of the two graphs $\left(N_{1}\right.$ and $\left.N_{2}\right)$, such that:

- $M($ uri $)=$ uri for each uri $\in U_{1} \cap N_{1}$

- $M($ lit $)=$ lit for each lit $\in L_{1}$

- $M$ maps bnodes to bnodes (i.e. for each $b \in B_{1}$ it holds $M(b) \in B_{2}$ )

- The triple $(s, p, o)$ is in $G_{1}$ if and only if the triple $(M(s), p, M(o))$ is in $G_{2}$.

It follows that if two graphs are equivalent then it certainly holds $U_{1}=U_{2}$, $L_{1}=L_{2}$ and $\left|B_{1}\right|=\left|B_{2}\right|$.

Let us now relate the problem of equivalence with edit distances.

\section{Def. 2 (Edit Distance over Nodes given a Bijection)}

Let $o_{1}$ and $o_{2}$ be two nodes of $G_{1}$ and $G_{2}$, and suppose a bijection between the nodes of these graphs, i.e. a function $h: N_{1} \rightarrow N_{2}$ (obviously $\left|N_{1}\right|=\left|N_{2}\right|$ ). We define the edit distance between $o_{1}$ and $o_{2}$ over $h$, denoted by $\operatorname{dist}_{h}\left(o_{1}, o_{2}\right)$, as the number of additions or deletions of triples which are required for making the "direct neighborhoods" of $o_{1}$ and $o_{2}$ the same (considering $h$-mapped nodes the same). Formally, $\operatorname{dist}_{h}\left(o_{1}, o_{2}\right)=$

$\left|\left\{\left(o_{1}, p, a\right) \in G_{1} \mid\left(o_{2}, p, h(a)\right) \notin G_{2}\right\}\right|+\left|\left\{\left(a, p, o_{1}\right) \in G_{1} \mid\left(h(a), p, o_{2}\right) \notin G_{2}\right\}\right|+$ $\left|\left\{\left(o_{2}, p, a\right) \in G_{2} \mid\left(o_{1}, p, h^{-1}(a)\right) \notin G_{1}\right\}\right|+\left|\left\{\left(a, p, o_{2}\right) \in G_{2} \mid\left(h^{-1}(a), p, o_{1}\right) \notin G_{1}\right\}\right| \diamond$

Now recall that if $G_{1}$ is equivalent to $G_{2}$ then there exists a bijection $h$ such that $(a, p, b) \in G_{1} \Leftrightarrow(h(a), p, h(b)) \in G_{2}$. We will denote this by $G_{1} \equiv_{h} G_{2}$. It follows that:

\section{Theorem 1 (RDF Graph Equivalence and Edit Distance)}

$G_{1} \equiv_{h} G_{2} \Leftrightarrow \operatorname{dist}_{h}(o, h(o))=0$ for each $o \in N_{1}$.

\footnotetext{
${ }^{1}$ A function that is both one-to-one (injective) and onto (surjective).
} 
Obviously the above theorem is useful for the case where the bijection $h$ respects the constraints of Def. 1 (i.e. maps named elements to named elements, and anonymous elements to anonymous).

\section{On Finding the Optimal Bnode Mapping}

Let us now focus on the case where two KBs, $K_{1}$ and $K_{2}$, are not necessarily equivalent and do contain bnodes. We would like to find a mapping over their bnodes that reduces the size (i.e. the number of change operations) of their delta and allows detecting whether $K_{1}$ is equivalent to $K_{2}$. Furthermore we want an efficient (tractable at least) method for finding such a mapping.

\subsection{RDF/S Differential Functions}

2122 described and analyzed various differential functions for comparing RDF/S knowledge bases. Each differential function returns a set of primitive change operations, i.e. $A d d(t)$ and $\operatorname{Del}(t)$ where $t$ is an RDF triple. For the needs of this paper, it is enough to use the differential function $\Delta_{e}$ which is defined as follows ("-" denotes set difference): $\Delta_{e}\left(K_{1} \rightarrow K_{2}\right)=\left\{A d d(t) \mid t \in K_{2}-K_{1}\right\} \cup\left\{\operatorname{Del}(t) \mid t \in K_{1}-K_{2}\right\}$. We call its output delta.

\subsection{Bnode Name Tuning and Delta Reduction Size}

The basic idea for reducing the delta is the following: if we match a bnode $b_{1}$ (of $B_{1}$ ) to a bnode $b_{2}$ (of $B_{2}$ ), through a bijection $M$, then these bnodes can be considered as equal at the computation of delta. For example, if $K_{1}$ contains a triple $\left(b_{1}\right.$, name, Joe $)$ and $K_{2}$ contains a triple $\left(b_{2}\right.$, name, Joe $)$ and we match $b_{1}$ to $b_{2}$, then these two triples will be considered equal and thus no difference will be reported. However we should note that in the context of versioning or synchronization services the change operations derived by a differential function should not be used as they are. For example, consider $K_{1}=\left\{\left(b_{1}\right.\right.$, name, Joe $\left.)\right\}$ and $K_{2}=\left\{\left(b_{2}\right.\right.$, name, Joe $),\left(b_{2}\right.$, lives, $\left.\left.U K\right)\right\}$ and suppose that we match again $b_{1}$ to $b_{2}$. In this case a mapping-aware comparison function will return the delta $\left\{A d d\left(\left(b_{2}\right.\right.\right.$, lives, $\left.\left.\left.U K\right)\right)\right\}$. If we want to apply it on $K_{1}$ then we have to replace $b_{2}$ by $b_{1}$, i.e we should apply on $K_{1}$ the operation $A d d\left(\left(b_{1}\right.\right.$,lives, $\left.\left.U K\right)\right)$, and in this way, we will obtain $K_{1}^{\prime}=\left\{\left(b_{1}\right.\right.$, name, Joe $),\left(b_{1}\right.$, lives, $\left.\left.U K\right)\right\}$ which is equivalent to $K_{2}$. We call this step Bnode Name Tuning, and it actually replaces (renames) in the delta the local names of the bnodes of $B_{2}$ by the local names of the matched bnodes in $B_{1}$. In this way the delta does not need any rename operation (i.e. rename $\left.\left(b_{1}, b_{2}\right)\right)$ and hence not any particular execution order.

Delta Reduction Size. Bnode matching cannot increase delta size. Without bnode matching any pair of bnodes from different KBs is considered different, and thus all triples to which they participate will be different and reported as 
change operations in the delta. On the other hand, if two bnodes are matched then the delta size is reduced if they participate to triples with the same predicate and the same other node (i.e. the same subject or object). In the case where all predicates/nodes of these triples are different, the delta size that will be reported is what will be reported without bnode matching.

\subsection{Bnode Matching as an Optimization Problem}

Here we formulate the problem of finding a mapping between the bnodes of two KBs as an optimization problem. Let $n_{1}=\left|B_{1}\right|, n_{2}=\left|B_{2}\right|$ and $n=\min \left(n_{1}, n_{2}\right)$. We have to match $n$ elements of $B_{1}$ with $n$ elements of $B_{2}$, i.e. our objective is to find the unknown part of the bijection $M$. To be more precise, $M$ a priori contains the mappings of all the URIs and literals of the KBs (URIs and literals are mapped as an identity function as in Def. 1), and its unknown part concerns $B_{1}$ and $B_{2}$. Suppose that $n=n_{1}<n_{2}$. Let $\mathcal{J}$ denote the set of all possible bijections between $B_{1}$ and a subset of $B_{2}$ that comprises $n$ elements. The number of all possible bijections (i.e. $|\mathcal{J}|)$ is $n_{2} *\left(n_{2}-1\right) * \ldots *\left(n_{2}-n_{1}+1\right)$, i.e. the first element of $B_{1}$ can be matched with $n_{2}$ elements of $B_{2}$, the second with $n_{2}-1$ elements, and so on. Consequently, the set of candidate solutions is exponential in size. Since our objective is to find a bijection $M \in \mathcal{J}$ that reduces the delta size (as regards the "unamed" parts of the KBs), we define the cost of a bijection $M$ as follows:

$$
\operatorname{Cost}(M)=\sum_{b_{1} \in B_{1}} \operatorname{dist}_{M}\left(b_{1}, M\left(b_{1}\right)\right)
$$

Def. 3 (The bijection yielding the less delta size) The best solution (or solutions) is defined as the bijection with the minimum cost, i.e. we define:

$$
M_{\text {sol }}=\arg _{M} \min _{M \in \mathcal{J}}(\operatorname{Cost}(M))
$$

The notation $\arg _{M}$ returns the $M$ in $\mathcal{J}$ that gives the minimum cost.

\section{Theorem 2 (Equivalence and Mapping Cost)}

If $G_{1} \equiv_{M_{s o l}} G_{2}$ (according to Def. 11) then $\operatorname{Cost}\left(M_{\text {sol }}\right)=0$.

The proof follows easily from the definitions. It is also clear that the inverse of Th. 2 does not hold (i.e. $\left.\operatorname{Cost}\left(M_{\text {sol }}\right)=0 \nRightarrow G_{1} \equiv_{M_{\text {sol }}} G_{2}\right)$ because the cost is based on the distance between the direct neighborhoods of the blank nodes only, and not between the named parts of the graphs.

From the algorithmic perspective, one naive approach for finding the best solution (i.e. $M_{s o l}$ ) would be to examine the set of all possible bijections. That would require at least $n$ ! examinations (true if $n_{1}=n_{2}=n$, while if $n_{1}<n_{2}$ then their number is higher than $n !)$. However, the problem is intractable in general:

Theorem 3. Finding the optimal bijection (according to Def. 3) is NP-Hard. 
Proof:

We will show that subgraph-isomorphism (which is NP-complete problem) can be reduced to the problem of finding the optimal bijection (meaning that our problem is at least as hard as subgraph-isomorphism). Let us make the hypothesis that we can find the optimal bijection in polynomial time. We will prove that if that hypothesis were true, then we would be able to solve the subgraph isomorphism in polynomial time. The subgraph isomorphism decision problem is stated as: given two plain graphs $G_{1}$ and $G_{2}$ decide whether $G_{1}$ is isomorphic to a subgraph of $G_{2}$. Let $G_{1}=\left(N_{1}, R_{1}\right)$ and $G_{2}=\left(N_{2}, R_{2}\right)$. We can consider these graphs as two RDF graphs such that: all of their nodes are bnodes and all property edges have the same label. Assume that $\left|N_{1}\right| \leq\left|N_{2}\right|$ and let $n=\min \left(\left|N_{1}\right|,\left|N_{2}\right|\right)$. If we can find in polynomial time whether there is a bijection between the $n$ nodes of $G_{1}$ and $n$ nodes of $G_{2}$ such that $\operatorname{Cost}\left(M_{\text {sol }}\right)=0$, then this means that we have found whether $G_{1}$ is isomorphic to a subgraph of $G_{2}$. Specifically, to decide whether there is a subgraph isomorphism, (a) we compute the optimal bijection, say $M_{\text {sol }}$, and (b) we compute its cost. If the cost returned by step (b) is 0 then we return YES, i.e. that there is a subgraph isomorphism. Otherwise we return NO (i.e. there is no subgraph isomorphism). Note that step (a) is polynomial by hypothesis, while step (b) relies on Def. Q 2 and its cost is again polynomial. Regarding the latter, note that $M_{\text {sol }}$ contains $n$ pairs, and to compute dist ${ }_{M}\left(b_{1}, b_{2}\right)$ for each $\left(b_{1}, b_{2}\right)$ pair of $M$, we consider only the direct neighborhoods of the two nodes in the two graphs (for $G_{2}$ we have to consider only those that connect nodes that participate in $M_{\text {sol }}$ 2. It follows that its computational cost is analogous to the number of edges of the graphs, and thus polynomial. Therefore given a bijection $M_{\text {sol }}$, to compute $\operatorname{Cost}\left(M_{\text {sol }}\right)$ requires polynomial time. Also note that Th. 1 holds also for plain graphs assuming a distance function over not labeled edges. We conclude that if our hypothesis were true, then we would be able to decide subgraph isomorphism in polynomial time.

We conclude that finding the optimal bijection is NP-Hard.

Below we will show that there are algorithms of polynomial complexity for a frequently occurring case. For the general case, we will propose algorithms of polynomial complexity that return an approximate solution.

\subsection{Polynomially-Solved (and Frequently Occurring) Cases}

Consider the KBs in Figure 2 and suppose that we want to compute $\operatorname{dist}_{h}\left(\right.$ - $_{\text {: }}$ $1,_{-}: 6$ ) (according to Def. 21). It is not hard to see that this distance depends on the mappings (by $h$ ) of the bnodes that are connected to $\_: 1$ and $\_: 6$, i.e. on the mappings of $\_: 3,_{-}: 4,_{-}: 8$ and $\_: 9$. However several datasets do not have directly connected bnodes. For this reason, here we study a variation of the problem that is appropriate for this case. The key point is that the distance between two bnodes does not depend on how the rest bnodes are mapped.

This is very important because in this case we can solve the optimization problem (as defined in Definition [3) using the Hungarian algorithm 12. (for short Alg $_{\text {Hung }}$, an algorithm for solving the assignment problem. Here the elements (bnodes) of $B_{1}$ play the role of workers, the elements (bnodes) of $B_{2}$ play

${ }^{2}$ Alternatively, if $\operatorname{Cost}\left(M_{\text {sol }}\right) \neq 0$ (using the distance as defined in the main paper), we return YES only if $\Delta_{e}\left(G_{1} \rightarrow G_{2}\right)$ as defined in section 3.1. after bnode name tuning, contains only triples each containing one bnode in $B_{1}$ and one not in $B_{1}$. 


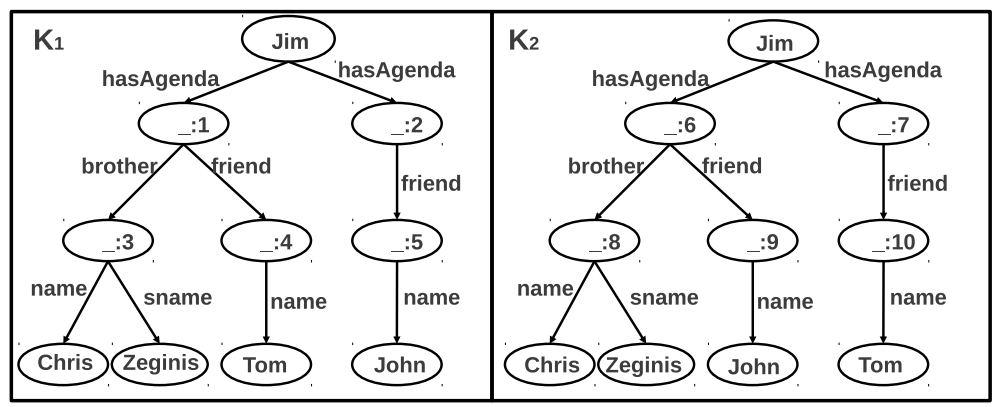

Fig. 2. Two KBs with directly connected bnodes

the role of jobs, and the edit distances of the pairs in $B_{1} \times B_{2}$ play the role of the costs. Consider for the moment that $\left|B_{1}\right|=\left|B_{2}\right|$. If we compute the edit distances between all possible $n^{2}$ pairs, then $A l g_{H u n g}$ can find the optimal assignment at the cost of $O\left(n^{3}\right)$ time. This means that finding the optimal solution costs polynomial time. An extension of $A g_{H}$ ung giving the ability to assign the problem in rectangular matrices (i.e. when $|B 1| \neq|B 2|$ ) is already provided in 2]. We conclude that if there are not directly connected bnodes then the optimal mapping can be found in polynomial time.

Theorem 4. Finding the optimal bijection (according to Def. 3) is a polynomial task if there are no directly connected bnodes.

\section{Bnode Matching Algorithms}

At section 4.1 we present a variation of $A l g_{H u n g}$ for getting an approximate solution for the general case, then at Section 4.2 we present a signature-based algorithm appropriate for larger datasets.

\subsection{Hungarian BNode Matching Algorithm}

We have already stated that $A \lg _{\text {Hung }}$ can find the optimal mapping in polynomial time if no directly connected bnodes exist in the compared KBs. For the cases where there are directly connected bnodes, $A g_{H}$ ung enriched with an assumption regarding how to treat the connected bnodes at the computation of dist $_{h}$, could be used for producing an approximate solution. Also in this case the algorithm will make $n_{1} \times n_{2}$ distance computations (where $n_{1}=\left|B_{1}\right|$ and $n_{2}=\left|B_{2}\right|$ ), and the complexity of the algorithm will be again $O\left(n^{3}\right)$.

Regarding connected bnodes, at the computation of $d i s t_{h}$, one could either assume that all of the connected bnodes are different, or all of them are the same. The first assumption does not require any bijection ( $h$ contains only the identity functions of the URIs and literals). According to Definition 2, the fact that all the bnodes are different means by extension that the triples in the direct 
neighborhoods connecting blank nodes are different too, even in the case where these triples have the same properties. For instance, applying the Definition 2 between bnodes $(-: 1, \ldots: 6)$ and $(-: 1, \ldots: 7)$ of Figure 2, we get that $\operatorname{dist}_{h}\left({ }_{-}: 1,,_{-}: 6\right)=4$ and $\operatorname{dist}_{h}\left({ }_{-}: 1,_{-}: 7\right)=3$ respectively. However, bnodes _ : $1, \ldots: 6$ have two outgoing triples with exactly the same properties, while bnodes _ : $1, \ldots$ : 7 have only one. We observe that this assumption is not very good because we would prefer $\_: 1$ to be "closer" to _ : 6 than to $\_: 7$.

According to the alternative assumption, when comparing bnodes $\left({ }_{-}: 1,{ }_{-}: 6\right)$ in Figure 2, bnode $\_: 3$ can be matched either with bnode $\_: 8$ or with bnode _ : 9, depending on the existence of a common property between them. This yields $\operatorname{dist}_{h}\left(-: 1_{-}: 6\right)=0$ since both bnodes have two outgoing triples with common properties (i.e. $\left({ }_{-}: 1\right.$, brother $\left.,_{-}: 3\right)$ is matched with $\left({ }_{-}: 6\right.$, brother, $\left.,_{-}: 8\right)$ and $\left({ }_{-}: 1\right.$, friend $\left._{-}: 4\right)$ is matched with $\left({ }_{-}: 6\right.$, friend $\left.\left._{-}: 9\right)\right)$. Regarding $\_: 1$ and _ : 7 , we get $\operatorname{dist}_{h}\left(-: 1_{-}: 7\right)=1$ because of the deleted triple $(-: 1$, brother,,$: 3)$. It follows that the results of this assumption are better over this example, as _ : 1 is "closer" to _ : 6 than to _ : 7. In general it is better because it exploits common properties, and therefore we adopt this assumption in our experiments.

\subsection{A Fast $(O(n \log N))$ Signature-Based Algorithm}

The objective here is to devise a faster mapping algorithm that could be applied to large KBs, at the cost of probably bigger deltas. We propose a signaturebased mapping algorithm, for short $A \lg _{\text {Sign }}$, which consists of two phases: the signature construction and the mapping construction phase. For each bnode $b$ we produce a string based on the direct neighborhood of $b$. This string is called the signature of bnode $b$. This phase gives us two lists of signatures, one for the bnodes of each KB. These lists should be considered as bags rather than sets, as there is a probability that two or more bnodes get the same signature. The probability depends on the way the signature is built (we discuss this later).

The mapping phase takes these two bags of strings and compares the elements of the first bag with those of the second. To make binary search possible, both

\begin{tabular}{|c|c|}
\hline $\begin{array}{l}\text { Alg. SignatureMapping } \\
\text { Input: two sets of bnodes } B_{1} \text { and } B_{2} \text {, } \\
\text { where }\left|B_{1}\right|<\left|B_{2}\right| \\
\text { Out: a bij. } M \text { between } B_{1} \text { and } B_{2} \\
\text { (1) } M=\emptyset \\
\text { (2) } B S_{1}=B S_{2}=\text { emptybag } \\
\text { (3) for each } b_{1} \in B_{1} \\
\text { (4) } B S_{1}=B S_{1} \cup\left\{\text { Signature }\left(b_{1}\right)\right\} \\
\text { (5) for each } b_{2} \in B_{2} \\
\text { (6) } B S_{2}=B S_{2} \cup\left\{\text { Signature }\left(b_{2}\right)\right\} \\
\text { (7) } \operatorname{sort}\left(B S_{1}\right) \\
\text { (8) } \operatorname{sort}\left(B S_{2}\right)\end{array}$ & 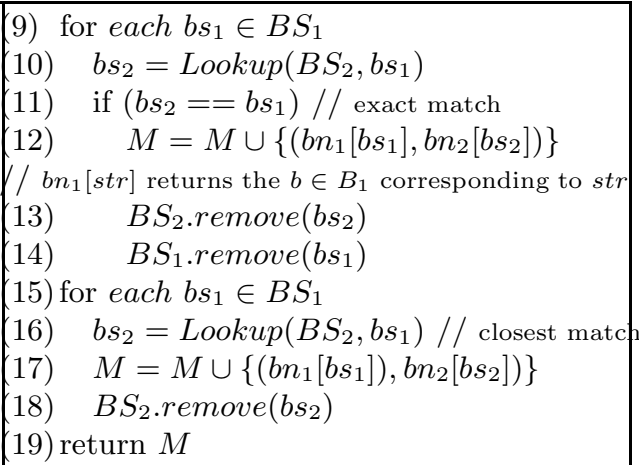 \\
\hline
\end{tabular}

Fig. 3. Alg. The Signature-based bnode matching algorithm 


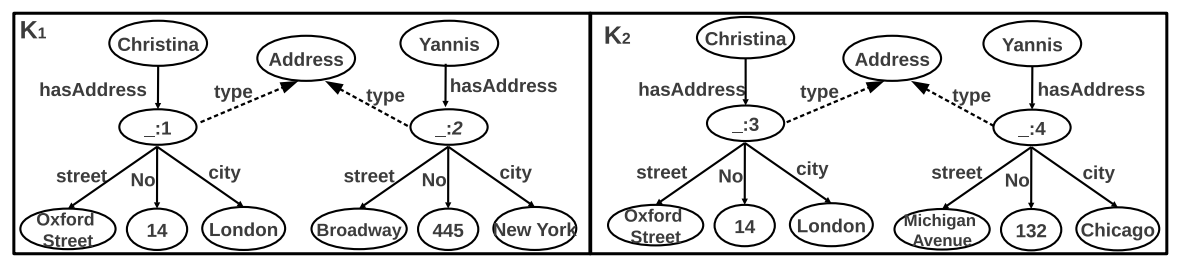

Fig. 4. Two versions of an address Knowledge Base

bags are sorted lexicographically. In particular, we start from the smaller list, say $B S_{1}$, and for each string $b s_{1}$ in that list we perform a lookup in the second list $B S_{2}$ using binary search. If an exact match exists (i.e. we found the string $b s_{1}$ also in $\left.B S_{2}\right)$ we produce a mapping, i.e. the pair $\left(b n_{1}\left[b s_{1}\right], b n_{2}\left[b s_{1}\right]\right)$. Since more than one bnodes may have the same signature we select one. We prefer the order as provided by the managing software, which in many cases reflects the order by which bnodes appear in files. As there is a high probability for subsequent versions to keep the same serialization, using the original order increases the probability of matches in case of same signatures. We continue in this way for all strings of $B S_{1}$. For each element $b s_{1}$ of $B S_{1}$ for which no exact match was found in $B S_{2}$ we perform a second lookup over the remainder part of $B S_{2}$, say $B S_{2}^{\prime}$, which will produce a mapping based on the closest element of $B S_{2}^{\prime}$ to the $b s_{1}$ element. Specifically, we will match $b s_{1}$ to the element of $B S_{2}^{\prime}$ to which binary search stopped, i.e. to the lexicographically closer element. Note that we perform the closest matches after finishing with the exact matches in order to avoid the situation where an approximate match deters an exact match at a later step.

The complexity of this algorithm is $O(n \log N)$ where $N=\max \left(n_{1}, n_{2}\right)$ and $n=\min \left(n_{1}, n_{2}\right)$, assuming that the average graph degree of bnodes (and thus signature size) does not depend on $N$. The algorithm is shown in Figure 3 and relies on an algorithm Signature for producing signatures, and on a algorithm Lookup as described earlier. As regards the signature construction method we would like to derive strings that allow matches that will yield small deltas even if the bnodes do not match exactly. To this end, we should give priority (i.e. bring to the front part of the string) to the items of the bnode that have lower probability to be changed from one version to the other.

Table 1. Signatures on bnodes of $K_{1}$ and $K_{2}$ of Fig. 4] according to the given option

\begin{tabular}{|c|c|}
\hline $\begin{array}{l}\text { Local } \\
\text { Name }\end{array}$ & Signature \\
\hline 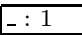 & ChristinahasAddress $\diamond$ typeAddress $\diamond$ cityLondon $*$ No14 $*$ streetOxfordStreet \\
\hline$-: 3$ & ChristinahasAddress $\diamond$ typeAddress $\diamond$ cityLondon $*$ No14 $*$ streetOxfordStreet \\
\hline-12 & YannishasAddress $\diamond$ typeAddress $\diamond$ cityNewYork $*$ No445* streetBroadway \\
\hline$-: 4$ & YannishasAddress $\diamond$ typeAddress $\diamond$ cityChicago $*$ No132 $*$ streetMichiganAvenue \\
\hline
\end{tabular}

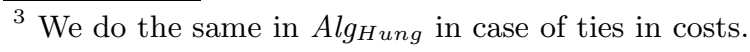


Consider bnode - : 1 of Figure 4 which is involved in the following triples: Incoming: $\left\{\left(\right.\right.$ Christina, hasAddress, $\left.\left.,_{-}: 1\right)\right\}$, Outgoing: $\{(-: 1$, street, Ox fordStreet $)$, (_: 1, No, 14), (_: 1, city,London) $\}$, Class Type: $\left\{\left(\_: 1,\right.\right.$ type, Address $\left.)\right\}$. Each of these triples will be mapped to a substring (e.g. "ChristinahasAddress" for the triple (Christina, hasAddress, _ : 1)). The set Class Type contains the triples with the rdf : type ("type" in the figure) property of the respective bnode. For the three different sets of triples (Incoming, Outgoing, Class Type) we are going to construct three sets of substrings respectively. The substrings inside each set are sorted lexicographically and separated by a special character, here denoted by $*$. The concatenation of these sets of substrings will yield the signature. A key point is the order by which the sets are concatenated. One option is to give a first priority to the set of the incoming triples, a second priority to the set with type information (i.e. "typeAddress"), and the last priority to the set of the outgoing triples. We should also mention that inside the signature the sets are separated by a special character, here denoted by $\diamond$. Table 1 shows the signatures of all the bnodes of Figure 4 according to this option. The proposed ordering of the substrings inside the signature stems from the assumption that the probability for the outgoing statements to change is higher than the incoming (e.g. in Figure 4 updating the address of a person is more probable than changing his/her name). Under this assumption the incoming statements should precede the outgoing inside the signature. Similarly for the class type of the bnode, it is not usual to be changed from one version to the other.

We represent the blank nodes which are subjects of incoming statements or objects of the outgoing statements, by a special character \& (i.e. we treat them as equal, as we did in the 2 nd assumption of approximation version of $A\left(g_{\text {Hung }}\right.$ ).

\section{$5 \quad$ Experimental Evaluation}

Real Datasets. We performed experiments for evaluating the potential for delta reduction, equivalence detection and time efficiency. In our experiments, we used two real datasets available in the LOD cloud: the Swedish open cultural heritage dataset5, and the Italian Museums dataset 6 , published from LKDI7. From each one we downloaded two versions with a time difference of one week or month. A preprocessing was necessary for corrections (e.g. missing URIs for some classes) and for merging the files. The features of these two datasets are given in Table 2, In both datasets there are no directly connected bnodes.

Experiments were conducted with and without bnode mapping. Regarding mapping we tested: (a) the random, (b) the Hungarian, and (c) the Signaturebased mapping methods. The results are shown in Table 3 . The first rows show

\footnotetext{
${ }^{4}$ Using Sesame RDF/S Repository (main memory), using a PC with Intel Core i3 at 2.2 Ghz, 3.8 GB Ram, running Ubuntu 11.10.

5 http://thedatahub.org/dataset/swedish-open-cultural-heritage used from http://kringla.nu/kringla/ for providing information on cultural data of Sweden.

6 http://thedatahub.org/dataset/museums-in-italy

7 http://www.linkedopendata.it/
} 
Table 2. Features of two real LOD datasets

\begin{tabular}{|l||l|l|l|l|}
\hline \multicolumn{1}{|c||}{} & \multicolumn{2}{c|}{ Swedish } & \multicolumn{2}{c|}{ Italian } \\
\hline & File 1 & File 2 & File 1 & File 2 \\
\hline \hline Date & $15 / 10 / 11$ & $22 / 10 / 11$ & $2 / 11 / 11$ & $4 / 12 / 11$ \\
\hline $\mid$ Triples $\mid$ & 3,750 & 3,589 & 49,897 & 49,897 \\
\hline $\mid$ BNodes $\mid$ & 535 & 509 & 6,390 & 6,390 \\
\hline Triples with bnodes $\mid$ & $77.7 \%$ & $77.2 \%$ & $43.85 \%$ & $43.85 \%$ \\
\hline Total Size & $378 \mathrm{~KB}$ & $365 \mathrm{~KB}$ & $5.49 \mathrm{MB}$ & $5.46 \mathrm{MB}$ \\
\hline
\end{tabular}

Table 3. Experimental results over real datasets

\begin{tabular}{|c|c|c|c|c|c|c|c|c|}
\hline & \multicolumn{4}{|c|}{ Swedish } & \multicolumn{4}{|c|}{\begin{tabular}{|c|} 
Italian \\
\end{tabular}} \\
\hline & without $B M$ & with B & M bnode & matching) & without $B M$ & with B & T(bnode & matching) \\
\hline & & Randor & $\operatorname{Alg}_{H u n g}$ & $\operatorname{Alg}_{\text {Sign }}$ & & Randor & $\operatorname{Alg}_{H u n s}$ & $\operatorname{Alg}_{\text {Sign }}$ \\
\hline|| $\mid$ Added $\mid$ & 2,805 & 2,726 & $\mid 75$ & 127 & 21,885 & 19,762 & 3 & 3 \\
\hline $\mid$ Deleted $\mid$ & 2,966 & 2,887 & 236 & 288 & 21,885 & 19,762 & 3 & 3 \\
\hline$\left|\Delta_{e}\right|$ & 5,771 & 5,613 & |311 & 419 & 43,770 & 39,524 & 6 & 6 \\
\hline BLoad Time $(\mathrm{ms})$ & 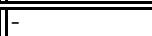 & 631 & 630 & 634 & $\overline{-1-}$ & 428 & 423 & $\overline{\mid 421}$ \\
\hline SC Time $(\mathrm{ms})$ & - & & & 210 & - & & & 840 \\
\hline BM Time $(\mathrm{ms})$ & - & 1.3 & 5,391 & 130 & - & 4.9 & 576,592 & 82.5 \\
\hline Diff Time $(\mathrm{ms})$ & 55 & 64 & 30 & 47 & 145 & 166 & \begin{tabular}{|l|l|}
169 \\
\end{tabular} & 163 \\
\hline Tuning Time $(\mathrm{ms})$ & & 15 & 0.2 & 0.5 & - & 3,332 & \begin{tabular}{|l|l|}
9.4 \\
\end{tabular} & 9.5 \\
\hline Total Time $(\mathrm{ms})$ & 57 & 715 & $\mid 5,931$ & 1,024 & 147 & 3,935 & 577,197 & 1,521 \\
\hline
\end{tabular}

the size of the yielded deltas and the last rows the time required for loading the bnodes (BLoad), constructing signatures (SC), bnode maping (BM), delta computation (Diff), bnode name tuning (Tuning Time), and the total time. We observe that the algorithms provide a delta of 12.7 to 7,294 times smaller than without bnode mapping. Alg $\lg _{H}$ y yields an equal (for the Italian) or smaller (0.34 times smaller for the Swedish) delta than $A \lg _{\text {Sign }}$, but it requires more time (from 15 to 624 times).

Synthetic Datasets. Although semantic data generators already exist in the bibliography, none of them deals with the blank node connectivity issues. Therefore we designed and developed a synthetic generator over the UBA (Univ-Bench Artificial data generator) [5] that can generate datasets with the desired bnode structures. Each dataset corresponds to an RDF graph $G$. Let $N$ odes be the set of all nodes in the graph, $B$ be the set of bnodes ( $B \subseteq$ Nodes), and conn $(o)$ be the nodes of $G$ that are directly connected with a node $o \in N o d e s$. We define $b_{\text {density }}$ as $b_{\text {density }}=\operatorname{avg}_{b \in B} \frac{|\operatorname{conn}(b) \cap B|}{|\operatorname{conn}(b)|}$. Note that if there are no directly connected bnodes then $b_{\text {density }}=0$. The extended generator can create datasets with the desired $b_{\text {density }}$ and the desired maximum length of paths that consist of edges that connect bnodes (we denote by $b_{\text {len }}$ their average). Using the synthetic generator, we created a sequence of 9 pairs of KBs (each pair has two subsequent versions of a $K B$ ). For instance, the first $\mathrm{KB}$ is $K_{0}$ and its pair is $K_{0}^{\prime}$. Each time we compare the subsequent versions of a pair with respect to mapping time and yielded delta size. From now on we express the delta size as a percentage of the number of triples of the KB, i.e. as $\frac{\left|\Delta_{e}\left(K, K^{\prime}\right)\right|}{\frac{|K|+\left|K^{\prime}\right|}{2}}$. Table 4 shows the blank node properties of each pair of KBs, its optimal delta size over its 
Table 4. Blank node Features of the synthetic dataset

\begin{tabular}{|c|c|c|c|c|c|c|c|}
\hline $\bar{K}$ & $\mid$ triples & $|B|$ & $\overline{D_{a}}$ & $b_{\text {density }}$ & $b_{l e n}$ & $\begin{array}{l}\text { Optimal } \\
\text { delta } \\
\text { size }\end{array}$ & Variation \\
\hline$\overline{K_{0 a}}$ & $\overline{5,846}$ & 240 & 13.4 & 0 & 0 & $1 \%$ & No connected blank nodes \\
\hline$\overline{K_{1 a}}$ & 5,025 & 240 & 10.5 & 0.1 & 1 & $0.5 \%$ & $\begin{array}{l}b_{-} \text {Neighborhoods of } 2 \text { bnodes, reduced } b_{-} \text {named } \\
\text { triples }\end{array}$ \\
\hline$\overline{K_{2 a}}$ & 2,381 & 240 & 7 & 0.15 & 1 & $1.5 \%$ & Reduced b_named triples \\
\hline$K_{3 a}$ & 1,628 & 240 & 5 & 0.2 & 1 & $1.5 \%$ & Reduced $b \_n a m e d$ triples \\
\hline $\bar{K}_{4 a}$ & 1,636 & 240 & 5 & 0.2 & 1.15 & $1 \%$ & $b_{-}$Neighborhoods of up to 8 bnodes \\
\hline$K_{5 a}$ & 1,399 & 240 & 4 & 0.25 & 1.15 & $1.7 \%$ & Reduced $b \_n a m e d$ triples \\
\hline$\overline{K_{6 a}}$ & 919 & 240 & 3 & 0.32 & 1.15 & $3.2 \%$ & 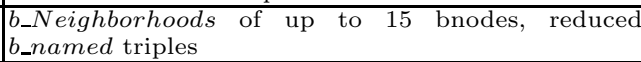 \\
\hline$\overline{K_{7 a}}$ & 909 & 240 & 3.25 & 0.4 & 1.35 & $2.7 \%$ & Connect $b \_N e i g h b o r h o o d s$, reduced $b \_n a m e d$ triples \\
\hline$\overline{K_{8 a}}$ & 1,001 & 240 & 3.94 & 0.5 & 21.5 & $2.5 \%$ & Connect $b_{-} N$ eighborhoods \\
\hline
\end{tabular}

subsequent version (known by construction) and its variation over the next pair of KBs (we call $b_{-} N e i g h b o r h o o d$ every subgraph having as nodes only bnodes, and we call $b \_n a m e d$ triple every triple that contains one bnode). With $D_{a}$ we denote the average number of direct edges of the bnodes (i.e. average number of triples to which a bnode participates).

Figure 5(left) gives the delta reduction potential of each algorithm in logarithmic scale. Without bnode mapping the delta size ranges from $95 \%$ (for the second pair of $\mathrm{KBs}$ ) to $143 \%$ (for the ninth pair of $\mathrm{KBs}$ ). Instead for Alg Hung it ranges from $0.47 \%$ to $10.67 \%$ and for $A_{\text {lgign }}$ it ranges from $1 \%$ to $11.5 \%$. Notice that $A \lg _{\text {Sign }}$ does not reduce the delta to the optimal for any pair of datasets, while $A l g_{\text {Hung }}$ achieves the optimal delta for most of the pairs.

Figure 5 (right) shows the delta reduction potential for the same pairs with the difference that the two bnode lists are not scanned in the original order (as in the left figure), but the second list is reversed. We notice that as the areas of directly connected bnodes become bigger (after the sixth pair of datasets), we get different (here higher) deltas. In such areas the direct neighborhoods lose their discrimination ability and thus the delta reduction potential becomes more unstable, increasing the probability to get a bigger delta.

If we use the optimal delta as baseline, and compute the percentage $\frac{\left|\Delta_{x}\right|-\left|\Delta_{\text {opt }}\right|}{\left|\Delta_{\text {opt }}\right|}$, in the first diagram this percentage for $A \lg _{H u n g}$ falls in $[0,2.88]$, while the Alg Sign $_{\text {'s }}$ percentage falls in $[0.4,3.2]$ (in the second diagram they fall in $[0,8]$ and $[0.4,8]$ resp.).
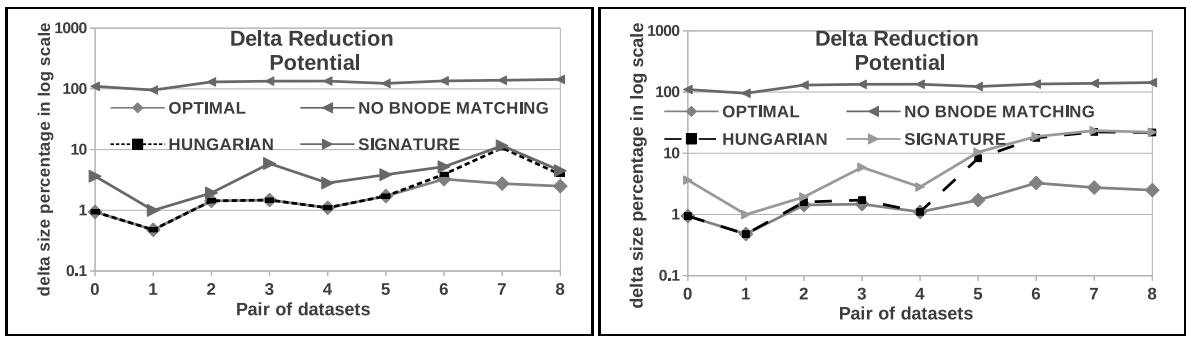

Fig. 5. Delta Reduction over the synthetic datasets 


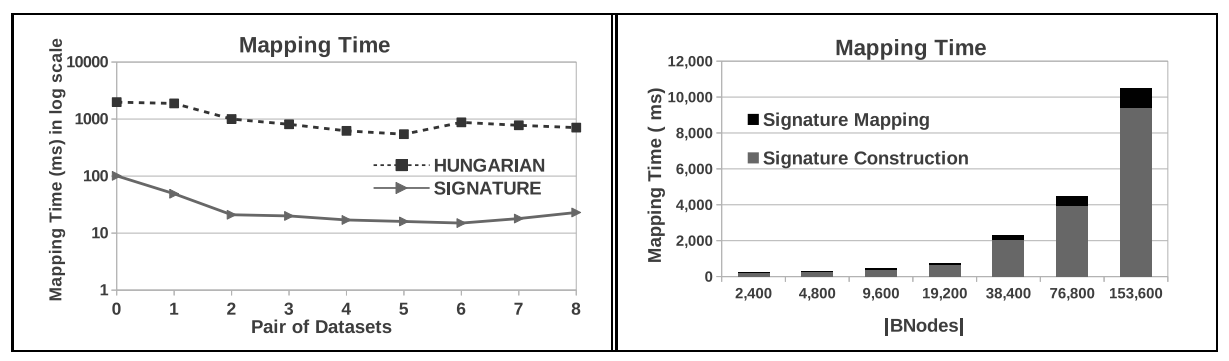

Fig. 6. Mapping times over the synthetic datasets

Figure 6(left) shows the mapping times of each algorithm in logarithmic scale. Alg $g_{\text {Sign }}$ gives two orders of magnitude lower mapping times.

Equivalences. Regarding equivalent $\mathrm{KBs}$, if there are no directly connected bnodes then $A l g_{\text {Hung }}$ detects them at polynomial time (recall Th. 4). To investigate what happens if there are directly connected bnodes we compared the pairs $(\mathrm{Kia}, \mathrm{Kia})$ for $\mathrm{i}=0$ to 8 of the synthetic KBs. In case of similarly ordered bnode lists both $A l g_{H u n g}$ and $A l g_{S i g n}$ detected equivalences for all the KBs, while for reverse scanned bnodes lists they detected 5 of the 9 equivalences. They did not detect equivalences for the KBs with $b_{\text {density }} \geq 0.25$.

Bigger Datasets. To investigate the efficiency of Alg $_{\text {Sign }}$ in bigger datasets, we created 7 pairs of KBs: the first pair contains 23,827 triples and 2,400 bnodes, the second pair has the double number of triples and bnodes, and so on, until reaching the last pair containing 153,600 bnodes. From Fig. 6 (right) we can see that the mapping time for $A l g_{\text {Sign }}$ was only 10.5 seconds for the seventh pair of

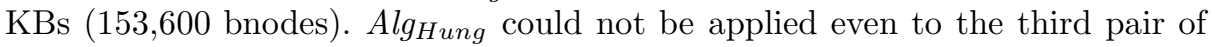
KBs due to its high (quadratic) requirements in main memory space.

The results are summarized in the concluding section.

Measuring the Approximation. The upper bound of the reduction of the delta size that can be achieved with bnode matching is the min number of bnodes of the two KBs multiplied by their average degree. Experimentally we have investigated whether $b_{\text {density }}$ (which is zero if there are no directly connected bnodes, and equal to 1 if all nodes are bnodes as in the proof of Th. 3), is related with the deviation from the optimal delta $d_{x}=\frac{\left|\Delta_{x}\right|-\left|\Delta_{o p t}\right|}{\left|\Delta_{o p t}\right|+1}$. Results over equivalent and non-equivalent KBs are shown at Figure 7 . Both algorithms give a much smaller deviation from optimal than without bnode matching (its $d_{x}$ ranges $[47,114]$ ). We also observe that keeping the original order of the bnodes is beneficial for both algorithms. For the non equivalent KBs the Alg Hung gives always equal or (mostly) smaller delta than the $A l g_{\text {Sign }}$, while for the equivalent both algorithms give exactly the same deviation. 


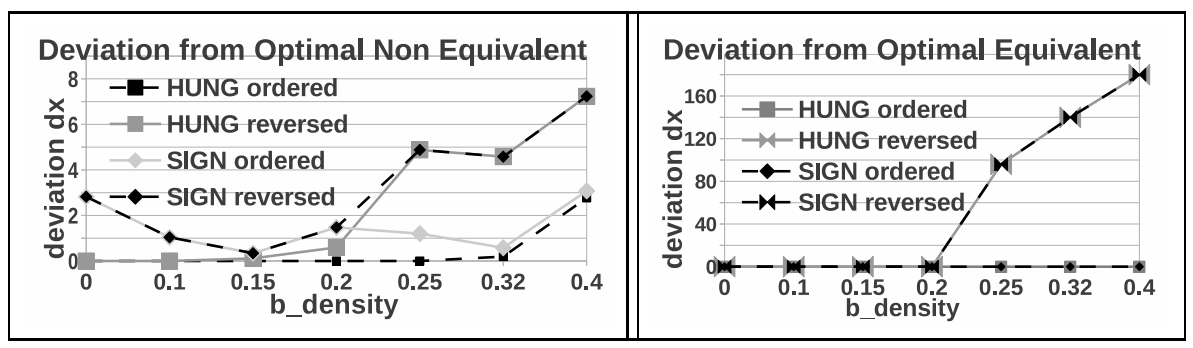

Fig. 7. $d_{x}$ over non equivalent (left) and equivalent (right) KBs

\section{Discussing Semantics and Inference Rules}

Apart from the explicitly specified triples of a KB, other triples can be inferred based on the RDF/S semantics 6], or other custom inference rules. In some cases one may want to decide whether two KBs are equivalent or to compute their delta with respect to a particular set of rules. In such scenarios, equivalence can be based again on the Def. 1 and the edit distance over nodes on the Def 2 with the only difference that the graphs should be completed according to the inferred triples. It follows that if the semantics is based on a set of inference rules yielding a finite closure, then the graph is finite and thus our method can be applied. Some semantics offering finite closures are RDF/S semantics, Minimal RDFS semantics [1], ter Horst's pD* semantics and OWL 2 RL, or even application-specific like [18].

It is worth mentioning, that the optimal bnode mapping over the complete graphs may be different from the optimal mapping when considering the explicit graphs. In the example of Figure 8, where fat arrows denote rdfs:subClass $0 f$ relationships and dotted arrows rdf :type relationships, the bijection with the minimum cost over the explicit graphs (left) is $\left\{\left(\_: 1, \_: 4\right),\left(\_: 2, \_: 3\right)\right\}$, while at the completed graphs (right) the bijection with the minimum cost is $\left\{\left(\_: 1, \_: 3\right),\left(\_: 2,-2: 4\right)\right\}$

Furthermore, for checking equivalence (at the presence of bnodes) or computing deltas, one could use the reduced graphs in case they are unique (note that the reduction of a $K_{a}$, is the smallest in size $K_{b}$ that is equivalent to $K_{a}$, i.e. $K_{a}$ and $K_{b}$ have the same closure).

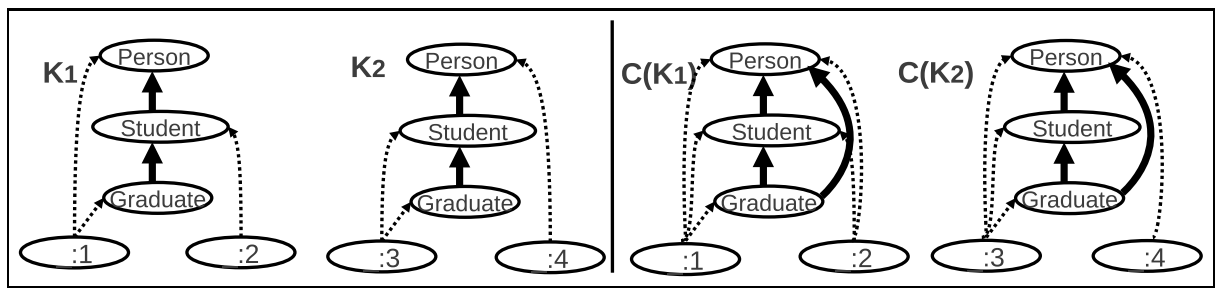

Fig. 8. Comparing the explicit versus the complete graphs of two KBs 


\section{Related Work}

Jena [3] provides a method for deciding whether two KBs that contain bnodes are equivalent (assuming Def. 1) and the adopted algorithm is GI-Complete. PromptDiff [16] and Ontoview [8] employ heuristic matchers to decide whether two bnodes from different KBs match or not, while CWM [1] is able to match two blank nodes only if they have functional term labels. Semversion 20] creates and assigns unique identifiers to bnodes so that to be able to identify the matching bnodes across versions. However, this is possible only if all versions have been derived from the same system. RDFSync [19] aims at fast synchronization, i.e. at reducing the parts of the KBs that have to be compared, and no effort is dedicated for finding a bnode mapping for reducing the delta size. [13] introduced a blank node mapping with $O\left(n^{2}\right)$ complexity aiming at merging sets of RDF triples (RDF molecules). However, this mapping presupposes that bnodes are parts of uniquely identified triples. This mapping method is not applicable in the general case and cannot be used for delta reduction. To the best of our knowledge our work is the first one that attempts to find a bnode mapping that reduces the size of deltas between KBs (that are not equivalent). Although there are several works for constructing RDF/S mappings (e.g. see [14]), they are not directly related since they map the named entities of the two KBs, and thus they take into account lexical similarities, something that is not possible with bnodes.

\section{Concluding Remarks}

In this paper we showed how we can exploit bnode anonymity to reduce the delta size when comparing $\mathrm{RDF} / \mathrm{S} \mathrm{KBs}$. We proved that finding the optimal mapping between the bnodes of two $\mathrm{KBs}$, i.e. the one that returns the smallest in size delta regarding the unnamed part of these KBs, is NP-Hard in the general case, and polynomial in case there are not directly connected bnodes. To cope with the general case we presented polynomial algorithms returning approximate solutions.

In real datasets with no directly connected bnodes $A \lg _{\text {Sign }}$ was two orders of magnitude faster than Alg $_{H u n g}$ (less than one second for KBs with 6,390 bnodes), but yielded up to 0.34 times (or $34 \%$ ) bigger deltas than $A l g_{H u n g}$, i.e. than the optimal mapping. $A l g_{H u n g}$ also identified all equivalent KBs.

For checking the behavior of the algorithms in KBs with directly connected bnodes, we created synthetic datasets, over which we compared $A \lg _{\text {Sign }}$ and the Alg $g_{\text {Hung }}$ approximation algorithm. Alg $g_{H u n g}$ yielded from 0 to 3 times smaller deltas than $A l g_{\text {Sign }}$, but the latter was from 18 to 57 times faster. Alg $\operatorname{lig}_{\text {Sign }}$ requires only 10.5 seconds to match 153,600 bnodes.

This is the first work on this topic. Several issues are interesting for further research. For instance, it is worth investigating other special cases where the optimal mapping can be found polynomially (e.g. directly connected bnodes that form graphs of bounded tree width). Another direction is to comparatively evaluate various (probabilistic) signature construction methods and greedy approximation algorithms. 
Software and datasets are available to download and use from: http://www.ics.forth.gr/isl/BNodeDelta.

Acknowledgement. Many thanks to the anonymous reviewers for their comments which helped us to improve the paper, as well to the members of FORTHICS-ISL. This work was partly supported by the NoE APARSEN (Alliance Permanent Access to the Records of Science in Europe, FP7, Proj. No 269977, 20112014), and the FP7 Research Infrastructures projects SCIDIP-ES (SCIence Data Infrastructure for Preservation - Earth Science, 2011, 2014), and iMarine (FP7 Research Infrastructures, 2011-2014).

\section{References}

1. Berners-Lee, T., Connoly, D.: Delta: An Ontology for the Distribution of Differences Between RDF Graphs (2004), http://www.w3.org/DesignIssues/Diff

2. Bourgeois, F., Lassalle, J.-C.: An extension of the Munkres algorithm for the assignment problem to rectangular matrices. Commun. ACM (1971)

3. Carroll, J.J.: Matching RDF Graphs. In: Horrocks, I., Hendler, J. (eds.) ISWC 2002. LNCS, vol. 2342, pp. 5-15. Springer, Heidelberg (2002)

4. Cloran, R., Irwin, B.: Transmitting RDF graph deltas for a Cheaper Semantic Web (2005)

5. Guo, Y., Pan, Z., Heflin, J.: LUBM: A benchmark for OWL knowledge base systems. Selected Papers from the Intern. Semantic Web Conf. ISWC (2004)

6. Hayes, P.: RDF Semantics, W3C Recommendation (2004)

7. Heflin, J., Hendler, J., Luke, S.: Coping with Changing Ontologies in a Distributed Environment. In: AAAI 1999 Workshop on Ontology Management (1999)

8. Klein, M., Fensel, D., Kiryakov, A., Ognyanov, D.: Ontology Versioning and Change Detection on the Web. In: Gómez-Pérez, A., Benjamins, V.R. (eds.) EKAW 2002. LNCS (LNAI), vol. 2473, pp. 197-212. Springer, Heidelberg (2002)

9. Klyne, G., Carroll, J.J.: Resource Description Framework (RDF): Concepts and Abstract Syntax (2004)

10. Mallea, A., Arenas, M., Hogan, A., Polleres, A.: On Blank Nodes. In: Aroyo, L., Welty, C., Alani, H., Taylor, J., Bernstein, A., Kagal, L., Noy, N., Blomqvist, E. (eds.) ISWC 2011, Part I. LNCS, vol. 7031, pp. 421-437. Springer, Heidelberg (2011)

11. Muñoz, S., Pérez, J., Gutierrez, C.: Simple and Efficient Minimal RDFS. Web Semantics (2009)

12. Munkres, J.: Algorithms for the assignment and transportation problems. JSIAM 5(1) (1957)

13. Newman, A., Li, Y.F., Hunter, J.: A scale-out RDF molecule store for improved coidentification, querying and inferencing. In: Intern. Workshop on Scalable Semantic Web Knowledge Base Systems, SSWS (2008)

14. Noessner, J., Niepert, M., Meilicke, C., Stuckenschmidt, H.: Leveraging Terminological Structure for Object Reconciliation. In: Aroyo, L., Antoniou, G., Hyvönen, E., ten Teije, A., Stuckenschmidt, H., Cabral, L., Tudorache, T. (eds.) ESWC 2010, Part II. LNCS, vol. 6089, pp. 334-348. Springer, Heidelberg (2010)

15. Noy, N.F., Kunnatur, S., Klein, M., Musen, M.A.: Tracking Changes During Ontology Evolution. In: McIlraith, S.A., Plexousakis, D., van Harmelen, F. (eds.) ISWC 2004. LNCS, vol. 3298, pp. 259-273. Springer, Heidelberg (2004) 
16. Noy, N.F., Musen, M.A.: PromptDiff: A Fixed-point Algorithm for Comparing Ontology Versions. In: Procs. of AAAI 2002 (2002)

17. Schandl, B.: Replication and Versioning of Partial RDF Graphs. In: Aroyo, L., Antoniou, G., Hyvönen, E., ten Teije, A., Stuckenschmidt, H., Cabral, L., Tudorache, T. (eds.) ESWC 2010, Part I. LNCS, vol. 6088, pp. 31-45. Springer, Heidelberg (2010)

18. Strubulis, C., Tzitzikas, Y., Doerr, M., Flouris, G.: Evolution of Workflow Provenance Information in the Presence of Custom Inference Rules. In: 3rd Intern. Workshop on the Role of Semantic Web in Provenance Management (SWPM 2012), co-located with ESWC 2012, Heraklion, Crete (2012)

19. Tummarello, G., Morbidoni, C., Bachmann-Gmür, R., Erling, O.: RDFSync: Efficient Remote Synchronization of RDF Models. In: Aberer, K., Choi, K.-S., Noy, N., Allemang, D., Lee, K.-I., Nixon, L.J.B., Golbeck, J., Mika, P., Maynard, D., Mizoguchi, R., Schreiber, G., Cudré-Mauroux, P. (eds.) ISWC/ASWC 2007. LNCS, vol. 4825, pp. 537-551. Springer, Heidelberg (2007)

20. Volkel, M., Winkler, W., Sure, Y., Ryszard Kruk, S., Synak, M.: SemVersion: A Versioning System for RDF and Ontologies. In: Procs. of ESWC 2005 (2005)

21. Zeginis, D., Tzitzikas, Y., Christophides, V.: On the Foundations of Computing Deltas Between RDF Models. In: Aberer, K., Choi, K.-S., Noy, N., Allemang, D., Lee, K.-I., Nixon, L.J.B., Golbeck, J., Mika, P., Maynard, D., Mizoguchi, R., Schreiber, G., Cudré-Mauroux, P. (eds.) ISWC/ASWC 2007. LNCS, vol. 4825, pp. 637-651. Springer, Heidelberg (2007)

22. Zeginis, D., Tzitzikas, Y., Christophides, V.: On Computing Deltas of RDF/S Knowledge Bases. ACM Transactions on the Web, TWEB (2011) 